\title{
Three dimensional transesophageal echocardiography guidance in closure of multihole atrial septal defect associated with patent foramen ovale
}

\author{
Zahra Khajali ${ }^{1}$, Ata Firouzi ${ }^{2}$, Maryam Hedayati $^{1}$, Azin alizadehas ${ }^{1}$, and Fatemeh Zohrian ${ }^{2}$ \\ ${ }^{1}$ Rajaie Cardiovascular Medical and Research Center \\ ${ }^{2}$ Rajaie Cardiovascular Medical \& Research Center, Tehran, Iran
}

May 6, 2020

\begin{abstract}
Treatment of patients with concomitant patent foramen ovale (PFO), atrial septal aneurysm (ASA), and multi fenestrated ASDs (mfASD) poses many challenges and may need several devices placed across the atrial septum which is more challenging, problematic and expensive. This is done by careful interrogation of the atrial septum by color doppler echocardiography, preferably by transoesophageal or three-dimensional transesophageal echocardiography (3D TEE) at the time of catheterization. We report one case who referred for PFO closures that were found to have an atrial septal aneurysm and multiple small holes. we used one occluder device Uni occlutech to close the PFO and fenestrations that this technique was with high success and without complication.
\end{abstract}

\section{Text:}

Treatment of patients with concomitant patent foramen ovale (PFO), atrial septal aneurysm (ASA), and multi fenestrated ASDs (mfASD) poses many challenges and may need several devices placed across the atrial septum which is more challenging, problematic and expensive. (1) It is important to have an accurate evaluation of the atrial septum to rule in or out the possibility of multiple defects. This is done by careful interrogation of the atrial septum by color doppler echocardiography, preferably by transoesophageal or three-dimensional transesophageal echocardiography (3D TEE) at the time of catheterization (2) As threedimensional echocardiography becomes more available, it will likely have a role in defining anatomy in preprocedure period as well. (3) We report one case who referred for PFO closures that were found to have an atrial septal aneurysm and multiple small holes. we used one occluder device Uni occlutech to close the PFO and fenestrations that this technique was with high success and without complication.

This patient was a 32-year-old lady with a history of percutaneous transcatheter pulmonary commissurotomy (PTPC) at age 20, chronic palpitation and dizziness referred to our clinic with recurrent symptoms of transient ischemic attack (left hemiplegia for 3 times) for doing the transesophageal echocardiography for evaluation of emboli source. Her general appearance was normal. The cardiac examination and ECG were unremarkable. The transesophageal echocardiography and three-dimensional transesophageal echocardiography showed normal left ventricle size and preserved systolic function (Ejection Fraction=50-55\%), normal right ventricle size with normal systolic function, normal biatrial size, normal drainage of all pulmonary veins to the left atrium, no clot or smoke in the left atrium and its appendage, no mitral stenosis, mild mitral regurgitation, normal and tricuspid aortic valve with no stenosis and insufficiency, thickened and dome shape pulmonary valve, no significant pulmonary stenosis ( Peak Pressure Gradient:18mmHg, Mean Pressure Gradient:10 mmHg), mild to moderate pulmonary insufficiency, mild tricuspid regurgitation, aneurysmal interatrial septum with large patent foramen oval (4 millimeters) and at least three fenestrations (larger one 
$=4$ millimeters) with left to right shunt with the distance between first and last hole was $12 \mathrm{~mm}$ and the nearest distance to PFO was $10 \mathrm{~mm}$ and most far distance is $24 \mathrm{~mm}$ (figure 1,2). The holes had sufficient rims but the septum was very aneurysmal. The present patient experienced a recurrent cerebrovascular attack at a relatively young age. A thorough investigation did not disclose any apparent etiology for this event other than a cryptogenic stroke due to a paradoxical embolus through the PFO or mfASD. The expected benefits for the present patient included the prevention of a recurrent stroke due to a presumed paradoxical embolus. So we scheduled the patient for PFO and multi fenestrated ASD device closure. We decided to perform percutaneous intervention but the most important factor was covering all of the defects. We selected a Uni device occlutech $33 \mathrm{~mm}$ for the procedure started with mild conscious sedation, there was aneurysmal interatrial septum, large patent foramen oval, and three small holes with sufficient all rims. During transcatheter closure, hemodynamic and saturation were obtained that the hemodynamic study showed normal blood pressure and heart rate with $\mathrm{O} 2$ saturation about 95\%. A Guidewire was advanced from a femoral vein, across one of the holes, into the left upper pulmonary vein under transesophageal echocardiography guidance. The tricky part of the procedure was passing the central hole for covering all fenestrations and Patent Foramen Ovale. The delivery sheath was then advanced over a wire, across the defect into the left atrium and left upper pulmonary vein, then closure device (Uni device occlutech, size $=33 \mathrm{~mm}$ ) was selected and advanced through the sheath into the left atrium, The left disc of the device was deployed with the catheter in the left atrium and then the catheter and device were withdrawn until the left disc gets parallel with the left atrium aspect of the interatrial septum. The right dick was then opened completely. At the first and second try, we could not obtain a good result and residual flow was seen. Finally, by guiding Three Dimensional Transesophageal Echocardiography we could pass the central fenestration (figure 3, movie 1) and the device covered all fenestrations and Patent Foramen Ovale and also aneurysmal part of the septum (figure 5). Before the device was released from the catheter, appropriate positioning was confirmed by interrogating all rims with echocardiography, and a wiggling maneuver was performed to confirm device stability)Movie 2 ). The benefits of using a single device are a shorter procedure duration and lesser chance of interference with venous blood flow, atrioventricular valves function, or adjacent tissue erosion (4). Then, after releasing of the device at the two weeks, two months, six months and one-year' follow-up visit, no complication was detected (no pericardial effusion, no compressive effect on contiguous structures such as an aortic valve, mitral and tricuspid valve, superior vena cava, inferior vena cava or pulmonary veins, no residual leakage even after contrast injection (movie3) and no recurrence of Transient Ischemic Attack). The immediate success rate of closure in this patient was very high due to the exact Three Dimensional Transesophageal Echocardiography and also due to the operator experience with this device. We empirically recommend the patient to receive antiplatelet agents (Aspirin and Plavix) for a period of at least 6 months to prevent any potential thromboembolic episodes.

\section{References:}

1. Numan, M. T., El-Said, H., Dilawar, M., \& El Sisi, A. The Challenge of Fenestrated Atrial Septal Defects closure; which device to choose?

2. Cao, Q-L., et al. "Transcatheter closure of multiple atrial septal defects. Initial results and value of two-and three-dimensional transoesophageal echocardiography." European heart journal21.11 (2000): 941-947

3. Tillman, Tywaun, Reynaldo Mulingtapang, and J. Thompson Sullebarger. "Approach to percutaneous closure in patients with multiple atrial septal defects." J Invasive Cardiol 20.5 (2008): E167-170

4. Tal, Roie, Qarawani Dahud, and Avraham Lorber. "Fenestrated atrial septal defect percutaneously occluded by a single device: procedural and financial considerations." Cardiology and therapy 2.1 (2013): $97-102$

\section{Figures:}

Figure 1 : Aneurysmal interatrial septum with large patent foramen oval and fenestration in Transesophageal Echocardiography. LA = left atrium, RA = right atrium, $\mathrm{RV}=$ right ventricle

Figure 2 : Aneurysmal interatrial septum and atrial septal fenestrations with left to right shunt in Trans- 
esophageal Echocardiography .LA = left atrium, RA = right atrium

Figure 3: Catheter passage from central hole in Three Dimensional Transesophageal Echocardiography

Figure 4: Uni device after releasing

Figure 5 : Uni device after releasing in Transesophageal Echocardiography. LA = left atrium, RA = right atrium

Movie 1: Three Dimensional Transesophageal Echocardiography showes catheter passage from a fenestration as a central hole

Movie 2: Wiggling maneuver was performed to confirm device stability before releasing

Movie 3: No bubble passage after device closure during contrast injection in transthoracic echocardiography. $\mathrm{LA}=$ left atrium; $\mathrm{LV}=$ left ventricle; $\mathrm{RA}=$ right atrium, $\mathrm{RV}=$ right ventricle

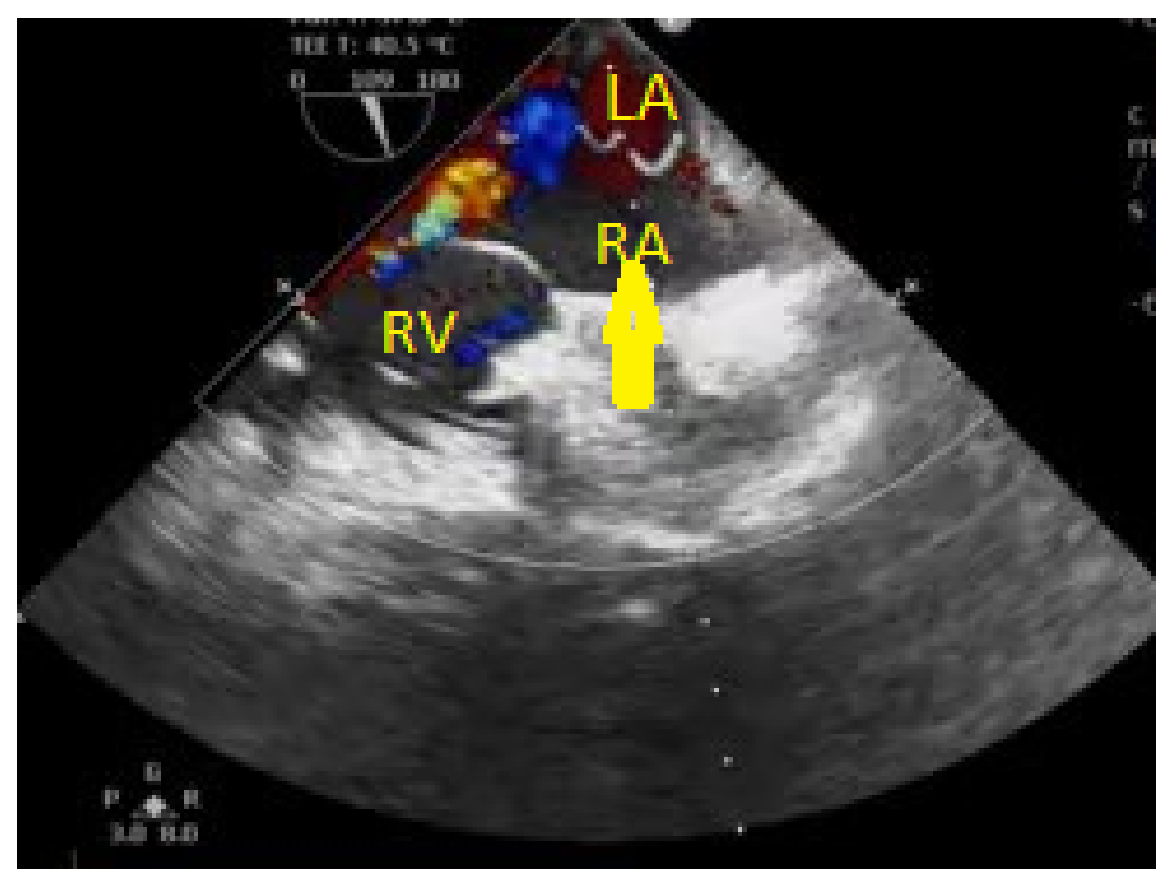



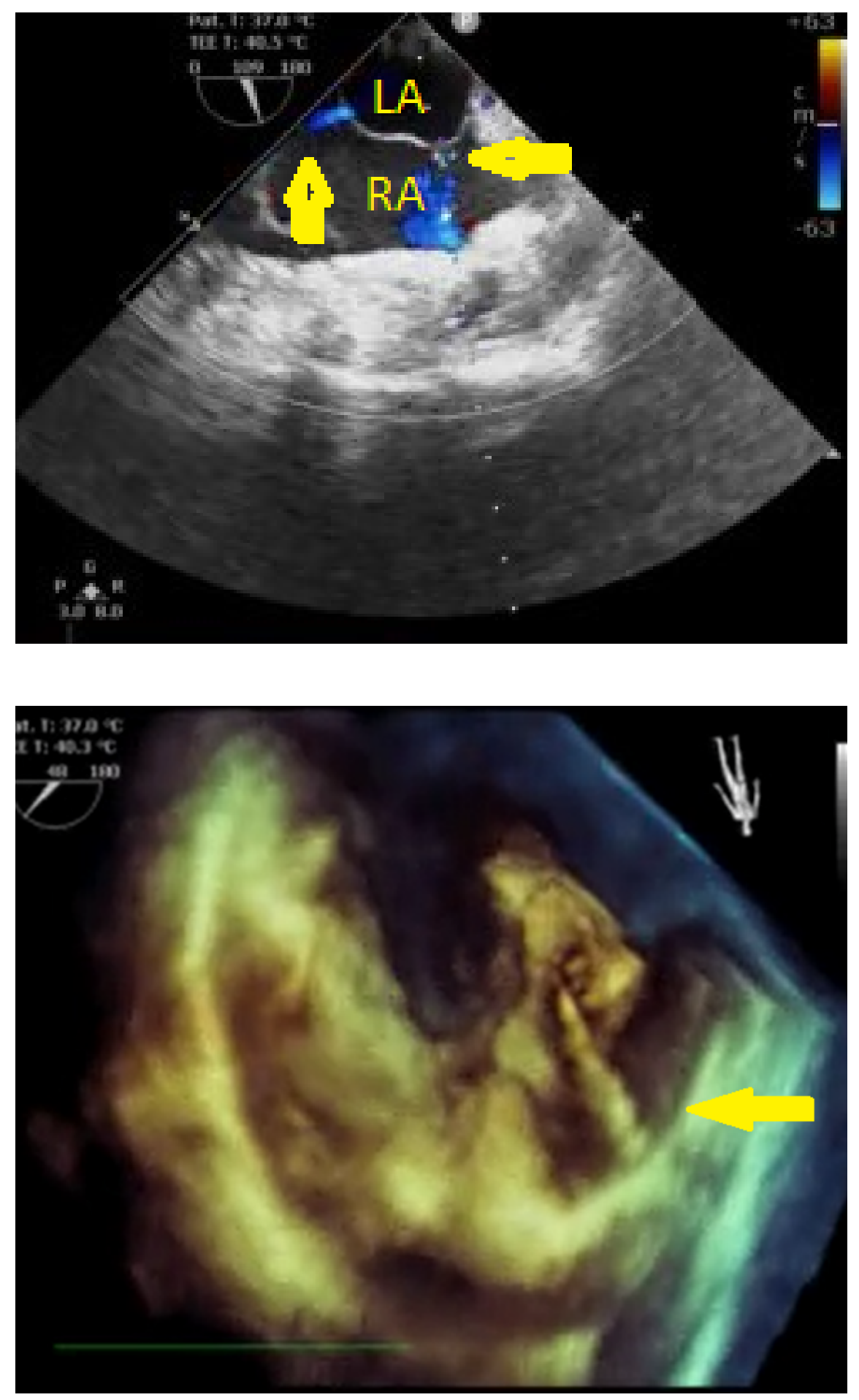


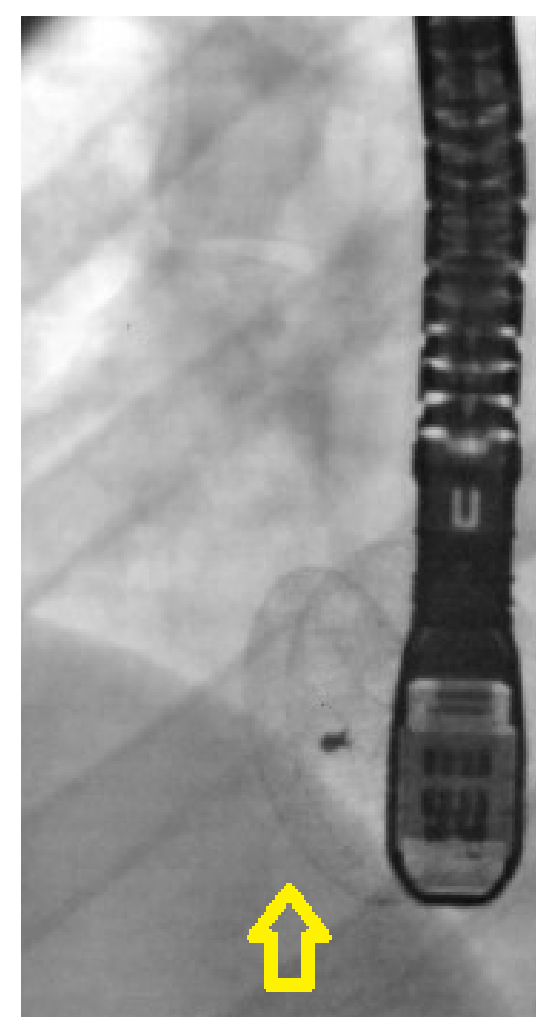




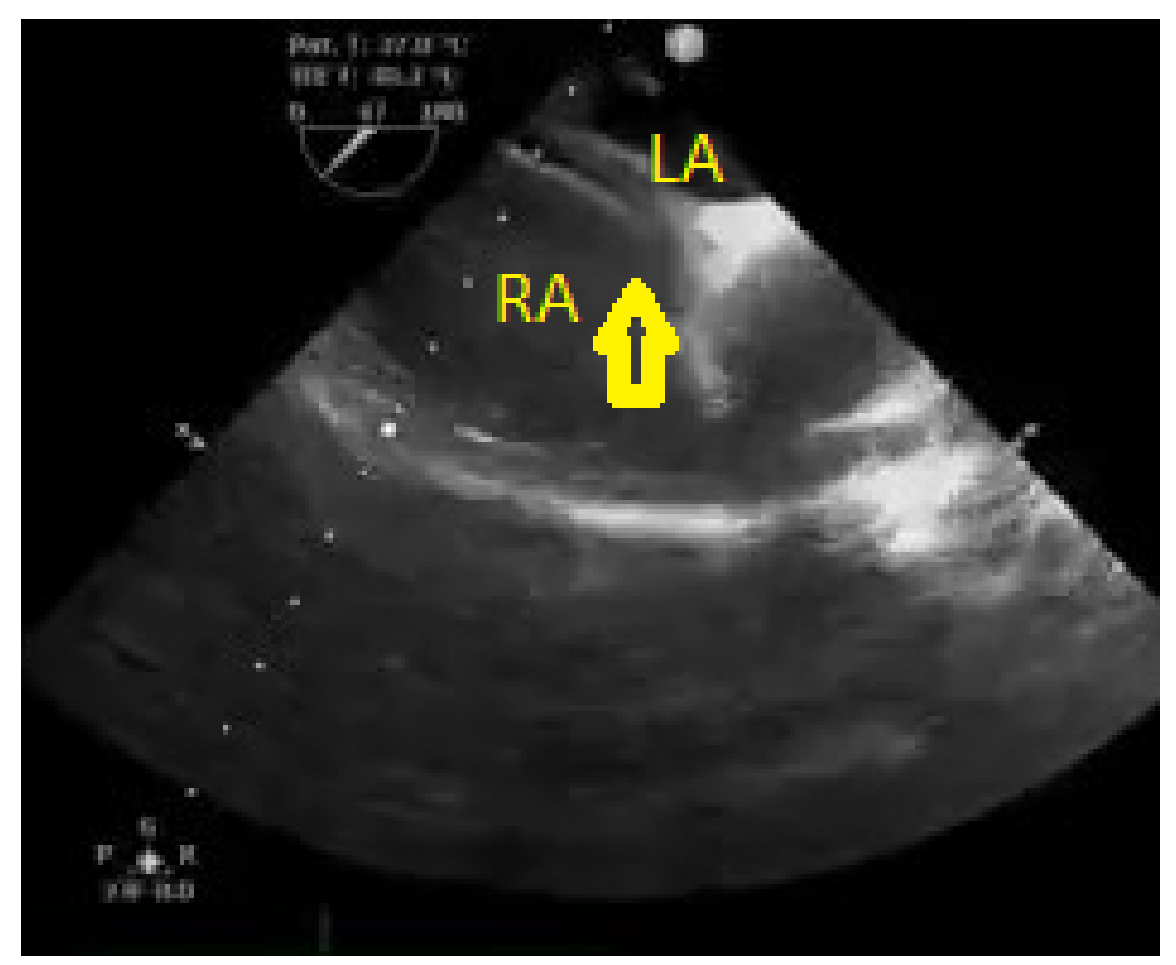

